TRABAJO ORIGINAL

\title{
EDUCACIÓN TERAPÉUTICA DE MUJERES CON DIABETES GESTACIONAL (EDUGEST): DATOS CORRESPONDIENTES AL PERÍODO DE RECLUTAMIENTO
}

\author{
EDUCATION OF PREGNANT WOMEN WITH GESTATIONAL DIABETES \\ (EDUGEST): DATA COLLECTED IN THE RECRUITMENT PERIOD
}

Silvia Gorbán de Lapertosa', Jorge Alvariñas², Jorge F. Elgart, Susana Salzberg ${ }^{4}$, Juan J. Gagliardino ${ }^{5}$

\begin{abstract}
RESUMEN
Introducción: la prevalencia de diabetes gestacional (DG) en Argentina es de 4,7\%, lo que implica mayor riesgo de desarrollar complicaciones tanto maternas como del recién nacido, prevenibles mediante un diagnóstico precoz y un tratamiento oportuno. Objetivos: promover la consulta precoz, el diagnóstico oportuno y el tratamiento adecuado mediante un programa de educación estructurado y multidisciplinario a nivel de Centros de Atención Primaria de la Salud (CAPs) y maternidades.

Materiales y métodos: se reclutaron embarazadas con DG en CAPs y maternidades de Buenos Aires, Corrientes, Chaco y Ciudad Autónoma de Buenos Aires (CABA), con la participación de obstetras, diabetólogos, enfermeras, asistentes sociales, educadores, nutricionistas y médicos comunitarios. Para el diagnóstico de DG se aplicaron los criterios de la Asociación Latinoamérica de Diabetes (ALAD) y se relevaron simultáneamente hábitos nutricionales y parámetros clínicos, metabólicos y terapéuticos. También se desarrollaron cursos de educación y seguimiento durante el embarazo, parto, postparto y lactancia. Resultados: las embarazadas con DG consultaron tardíamente (la mayoría en el tercer trimestre de embarazo) y presentaron factores de riesgo para DG no prevenibles (el más frecuente fue el antecedente familiar de diabetes) y prevenibles (obesidad, hipertrigliceridemia y macrosomía) en embarazos previos.

Conclusiones: el EduGest pretende interrumpir el círculo vicioso generado por la DG sobre la futura diabetes mellitus tipo 2 en la madre y el niño al promover la adopción de hábitos saludables.
\end{abstract}

Palabras clave: diabetes gestacional; obesidad; hipertrigliceridemia; educación; hábitos saludables; prevención.

\begin{abstract}
Introduction: in Argentina the prevalence of gestational diabetes $(G D)$ is $4.7 \%$, accounting for a higher risk of developing complications in both the mother and the baby which could be prevented by early diagnosis and timely treatment.

Objectives: to promote early consultation, timely diagnosis and adequate treatment through a structured and multidisciplinary education Program in Primary Healthcare (PHU) and maternity (PMU) units.
\end{abstract}

Materials and methods: pregnant women with DG were recruited in PHU and PMU from Buenos Aires, Corrientes, Chaco and CABA, Argentina. The program included the participation of obstetricians, diabetologists, nurses, social workers, educators, nutritionists and community doctors. The ALAD diagnostic criteria for DG were applied. Simultaneously, nutritional habits, clinical, metabolic and therapeutic parameters were recorded. Education and follow-up courses were developed during pregnancy, delivery, after delivery and breastfeeding.

Results: late consultation was frequent in pregnant women with GD (mainly during the third trimester), who presented both non-preventable (the most frequent being family history of diabetes) and preventable (obesity, hypertriglyceridemia and macrosomia) risk factors for GD in previous pregnancies.

Conclusions: EduGest is aimed at breaking the vicious circle of $D G$ on the future development of type 2 diabetes in the mother and the baby by promoting the adoption of healthy habits.

Key words: gestational diabetes; obesity; hypertriglyceridemia; education; healthy habits; prevention.
Profesora Titular de Nutrición y Educación para la Salud, Carrera de Licenciatura en Kinesiología y Fisiatría, Facultad de Medicina, Universidad Nacional del Nordeste (UNNE), Corrientes, Argentina

2 Médico Consultor, Hospital Gral. de Agudos Enrique Tornú, Ciudad Autónoma de Buenos Aires, Argentina

3 Investigador Adjunto del Consejo Nacional de Investigaciones Científicas y Técnicas (CONICET), Centro de Endocrinología Experimental y Aplicada (CENEXA; Universidad Nacional de La Plata -UNLP-, CONICET, Comisión de Investigaciones Científicas de la Provincia de Buenos Aires -CICPBA-), Facultad de Ciencias Médicas, Universidad Nacional de la Plata, La Plata, Provincia de Buenos Aires, Argentina

4 Directora del Departamento de Investigaciones Clínicas, Instituto Centenario, Ciudad Autónoma de Buenos Aires, Argentina
Investigador Superior Emérito del Consejo Nacional de Investigaciones Científicas y Técnicas (CONICET), Centro de Endocrinología Experimental y Aplicada (CENEXA; Universidad Nacional de La Plata -UNLP-, CONICET, Comisión de Investigaciones Científicas de la Provincia de Buenos Aires -CICPBA-), Profesor Consulto de la Facultad de Ciencias Médicas de la Universidad Nacional de La Plata, La Plata, Provincia de Buenos Aires, Argentina

\footnotetext{
Contacto de la autora: Silvia Beatriz Gorbán de Lapertosa E-mail: dralapertosa@hotmail.com Correspondencia: Facultad de Medicina UNNE, Moreno 1240 (3400), Corrientes, Argentina Fecha de trabajo recibido: 12/10/19 Fecha de trabajo aceptado: 13/11/19
}

Conflictos de interés: los autores declaran que no existe conflicto de interés 


\section{INTRODUCCIÓN}

La diabetes gestacional (DG) se define como una intolerancia a la glucosa que se manifiesta durante el embarazo ${ }^{1-3}$. Empleando el criterio diagnóstico de la Asociación Latinoamericana de Diabetes (ALAD), su prevalencia en Argentina es de 4,7\% ${ }^{4}$.

Las mujeres con DG presentan mayor riesgo de desarrollar complicaciones durante su embarazo (preeclampsia), mientras que sus hijos tienen un mayor riesgo de desarrollar eventos adversos a corto plazo (macrosomía, hipoglucemia neonatal y disfunción cardíaca neonatal), así como disfunciones metabólicas a largo plazo, tales como obesidad, tolerancia a la glucosa alterada (TGA) y diabetes en la adolescencia o en la adultez temprana ${ }^{1,5,6}$.

Además de las alteraciones inducidas en el feto y la madre durante el embarazo, ambos también son afectados después del parto. En Estados Unidos, entre el 5 y el $10 \%$ de las mujeres con DG desarrollan diabetes mellitus tipo 2 (DM2) inmediatamente después del embarazo 5 . Aún cuando la DG revierte dentro de las seis semanas posteriores al parto, aproximadamente el $50 \%$ de las mujeres con este diagnóstico desarrollaría DM2 en los próximos 10 a 30 años?

El impacto negativo sobre la madre y el recién nacido puede reducirse significativamente mediante un diagnóstico precoz y un tratamiento adecuado de la DG, que combine la adopción de un estilo de vida saludable y medicación ${ }^{8,9}$. En función de las evidencias mencionadas, recientemente implementamos en nuestro país un programa (EduGest) que intenta disminuir la carga de la enfermedad.

El objetivo principal del EduGest es promover una acción conjunta de Centros de Atención Primaria de la Salud (CAPs) y maternidades de Argentina para facilitar el acceso de las pacientes a una consulta precoz, un diagnóstico oportuno y un tratamiento adecuado mediante un programa de educación estructurado y multisectorial. En esta ocasión presentamos su diseño y los datos registrados durante el período de reclutamiento de las participantes.

\section{MATERIALES Y MÉTODOS}

Las embarazadas con DG identificadas en los CAPs y las maternidades participantes durante el período 2017-2019 fueron derivadas para recibir atención médica especializada. Para ello previamente establecimos una red que incluyó las si- guientes instituciones: Hospitales J.R. Vidal y A.I de Llano de Corrientes, Hospital Zonal Camilo Muniagurria de Goya, Hospital Dr. Fernando Irastorza de Curuzu Cuatiá, y CAPs y Salas Municipales (SAPs) de Corrientes; Hospital Dr. J.C. Perrando de Chaco; Hospitales C. Argerich, J. Ramos Mejía y Donación F. Santojanni de la Ciudad Autónoma de Buenos Aires (CABA); Hospital Municipal Materno Infantil de San Isidro y Hospital Diego Thompson de San Martín, provincia de Buenos Aires. Las pacientes derivadas de los diferentes centros reclutados siguieron el circuito que se muestra en la Figura 1.

A lo largo de este circuito, el equipo de atención médica completó para cada participante el formulario QualiDiabGest específicamente desarrollado para este estudio. El mismo incluye datos personales y antecedentes obstétricos, índice de masa corporal (IMC), presión arterial y factores de riesgo cardiovascular (FRCV). También se implementó el formulario de bienestar OMS-5 con la puntuación de Lowe para identificar depresión ${ }^{10,11}$. Además se extrajeron muestras de sangre para determinar glucemia en ayunas, colesterol total y nivel de triglicéridos (TG). Las determinaciones de laboratorio se realizaron con kits comerciales.

\section{Análisis estadístico}

Los datos registrados durante la consulta habitual se almacenaron en forma anonimizada en la base de datos QualiDiabGest para su posterior análisis con el paquete estadístico para Ciencias Sociales versión 15 (SPSS Inc., Chicago, IL, EE.UU.). Los resultados de estadísticas descriptivas para variables continuas se presentaron como medias \pm desvíos estándar (DE), mediana y rango intercuartílico $(\mathrm{R} \mid \mathrm{Q})$; para las variables categóricas se presentaron como proporciones con $95 \%$ de intervalo de confianza (IC) según correspondiera.

\section{Consideraciones éticas}

El protocolo de estudio fue analizado y aprobado por el Comité de Ética de la Universidad Nacional del Nordeste (UNNE). Todos los procedimientos del estudio también se ajustaron a los estándares éticos de la Declaración de Helsinki de 1964 y sus modificaciones posteriores, o estándares éticos comparables. Todas las participantes incluidas en el estudio firmaron su consentimiento informado. 


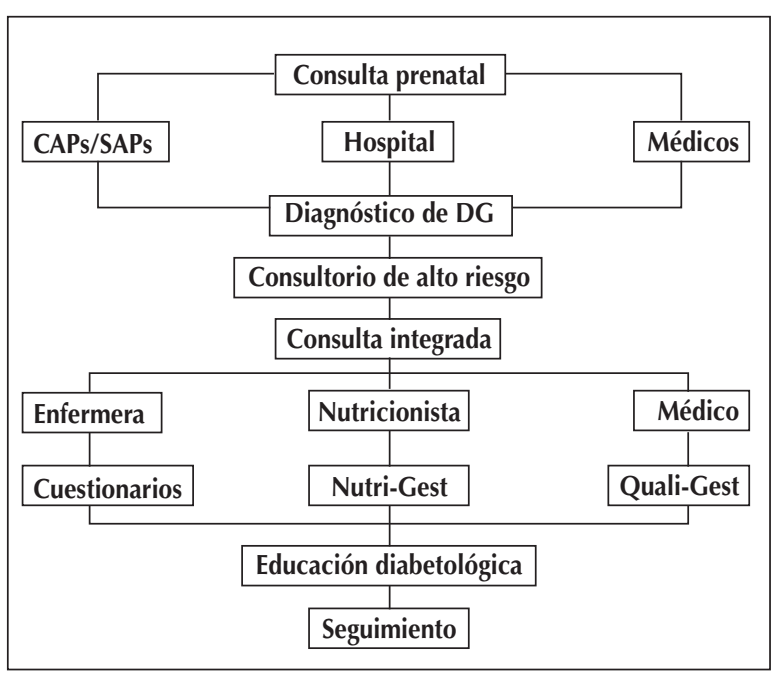

Figura 1: Flujo de pacientes del EduGest.

\section{RESULTADOS}

El programa educativo constó de un conjunto de componentes dirigidos a diferentes audiencias, a saber:

1. Comunidad

- Campaña de sensibilización mediante la cual se difundió información acerca de la frecuencia y gravedad de la DG, su impacto sobre la madre y el producto de la gestación y cómo diagnosticarla, prevenir su manifestación y tratarla adecuadamente.

2. Médicos y enfermeras

- Capacitación específica en el área de DG.

- Capacitación sobre normas de diagnóstico, prevención y tratamiento. La misma consistió en una jornada teórico-práctica intensiva de un día de duración donde especialistas del área de diabetes y obstetricia desarrollaron los contenidos de las Guías de diagnóstico y tratamiento de diabetes gestacional ALAD 2016². La jornada se desarrolló en todas las áreas programáticas del EduGest (Corrientes, Chaco, CABA y provincia de Buenos Aires).

\section{Educadores}

- Capacitación y provisión de material educativo para el dictado de cursos estructurados de modalidad grupal e interactiva para embarazadas con DG.

4. Embarazadas con DG

- Cursos estructurados en pequeños grupos para embarazadas con DG y sus familiares. Para el desarrollo de estas actividades se utilizaron diferentes materiales: trípticos y afiches, presentaciones Power Point para su empleo en los cursos interactivos, tarjetas con fotos de alimentos y código de señales de tránsito según su valor calórico, modelo de periné y canal vaginal para simulación del parto, muñeco para práctica de lactancia materna, modelos de métodos anticonceptivos, modelo para prácticas de autoinyección de insulina, jeringas y plumas para autoinyección de insulina, tiras reactivas y diferentes glucómetros para práctica de automonitoreo glucémico (AMG), cuadernillos para registro diario de valores de AMG y el manual: "Cómo tratar mi diabetes gestacional," que contiene los conceptos presentados en el curso presencial interactivo.

El programa educativo incluye un registro sistemático de datos que permite: 1) realizar un diagnóstico de la situación real del problema (DG) en nuestro medio, y 2) evaluar el impacto del programa educativo desde el punto de vista de la prevención y el control de esta patología. Dicho registro utiliza los siguientes formularios:

- QualiDiabGest (registro clínico-metabólico y de eventos gestacionales correspondientes a la madre y al feto/recién nacido).

- NutriQuidGest (autoevaluación de la ingesta alimentaria con un programa informático que transforma los alimentos en sus componentes esenciales) $)^{13}$.

- OMS-5 con puntaje de Lowe, que evalúa bienestar y simultáneamente permite determinar la tendencia a la depresión ${ }^{10,11}$.

El contenido detallado de cada material descripto puede consultarse en el sitio oficial de la Facultad de Medicina de la Universidad Nacional del Nordeste ("Proyectos Institucionales": http:// cv.med.unne.edu.ar/course/view.php?id=1556).

En la etapa de reclutamiento de embarazadas con DG registramos 1.468 pacientes, cuyos datos se muestran en la Tabla 1. Puede apreciarse que la edad media fue $30,9 \pm 6,4$ años, con $2,2 \pm 1,9$ gestas previas y una edad gestacional de $28,8 \pm 6,5$ semanas.

Entre los antecedentes personales, el 55,3\% de las mujeres embarazadas con DG tenía historia familiar de diabetes e IMC de aproximadamente $30 \mathrm{~kg} / \mathrm{m}^{2}$, mientras que los valores de presión arterial al igual que los de glucemia en ayunas se ubicaron dentro de los límites normales. Por el contrario, los valores de colesterol total y TG estuvieron por encima de los valores de corte normal para la etapa de gestación (tercer trimestre) de nuestra población ${ }^{14}$.

Entre los antecedentes obstétricos patológicos, el más frecuente fue haber tenido niños con peso al nacer $\geq 4 \mathrm{~kg}(18 \%)$, seguido de presencia de DG en embarazos previos $(14,4 \%)$, hipertensión inducida por el embarazo (HIE 9,1\%), prematuridad fetal $(8,9 \%)$, preeclampsia $(6,6 \%)$ y eclampsia $(1,1 \%)$ (Tabla 2$)$. 


\begin{tabular}{|l|c|c|c|c|c|}
\hline \multicolumn{1}{|c|}{ Parámetro } & Media & DS & Mediana & RIQ & n \\
\hline Edad al inicio del embarazo (años) & 30,9 & 6,4 & 31 & $(26-36)$ & 1.432 \\
\hline Gestas previas (cantidad) & 2,2 & 1,9 & 2 & $(1-3)$ & 1.411 \\
\hline Edad gestacional (semanas) & 28,8 & 6,5 & 30 & $(26-34)$ & 1.166 \\
\hline Talla $(\mathrm{cm})$ & 158,6 & 6,5 & 158 & $(154-163)$ & 1.267 \\
\hline Peso al inicio $(\mathrm{kg})$ & 75,4 & 18,0 & 74 & $(62-85)$ & 1.296 \\
\hline IMC $\left(\mathrm{kg} / \mathrm{m}^{2}\right)$ & 29,8 & 6,6 & 29 & $(25-34)$ & 1.176 \\
\hline TAS (mmHg) & 107,9 & 13,9 & 110 & $(100-120)$ & 1.326 \\
\hline TAD $(\mathrm{mmHg})$ & 66,6 & 9,8 & 60 & $(60-70)$ & 1.323 \\
\hline Glucemia en ayunas $(\mathrm{mg} / \mathrm{dl})$ & 98,3 & 29,1 & 93 & $(82-105)$ & 1.191 \\
\hline Colesterol total $(\mathrm{mg} / \mathrm{dl})$ & 230,0 & 56,1 & 225 & $(192-263)$ & 688 \\
\hline TG $(\mathrm{mg} / \mathrm{dl})$ & 231,2 & 94,0 & 218 & $(167-2739$ & 583 \\
\hline
\end{tabular}

DS: desvío estándar; RIQ: rango intercuartílico; IMC: índice de masa corporal; TAS: tensión arterial sistólica; TAD: tensión arterial diastólica; TG: triglicéridos.

Tabla 1: Características generales de la muestra.

\begin{tabular}{|l|l|l|l|l|}
\hline \multicolumn{1}{|c|}{ FRCV recurrentes } & \multicolumn{1}{c|}{$\mathbf{n}$ n (Sí) } & \multicolumn{1}{c|}{ \% } & \multicolumn{1}{c|}{ (IC 95\%) } \\
\hline Hipertensión crónica & 1.468 & 86 & 5,9 & $(4,7-7,1)$ \\
\hline Obesidad & 1.468 & 385 & 26,2 & $(24,0-28,5)$ \\
\hline Dislipemia & 1.468 & 28 & 1,9 & $(1,2-2,6)$ \\
\hline Tabaquismo & 1.468 & 95 & 6,5 & $(5,2-7,7)$ \\
\hline \multicolumn{1}{|c|}{ Antecedentes obstétricos } & & 180 & & \\
\hline DG en gestas previas & 1.252 & 117 & 14,4 & $(12,4-16,3)$ \\
\hline Prematuridad fetal & 1317 & 87 & 8,9 & $(7,3-10,4)$ \\
\hline Preeclampsia & 1310 & 759 & 6,6 & $(5,3-8,0)$ \\
\hline $\begin{array}{l}\text { Antecedentes de familiares con } \\
\text { diabetes }\end{array}$ & 1372 & 244 & 55,3 & $(52,7-58,0)$ \\
\hline Nacidos con peso $>4$ kg & 1353 & 120 & 18,0 & $(16,-20,1)$ \\
\hline HIE en gestas previas & 1314 & 15 & 9,1 & $(7,6-10,7)$ \\
\hline Eclampsia & 1316 & 1,1 & $(0,6-1,7)$ \\
\hline
\end{tabular}

FRCV: factor de riesgo cardiovascular; IC: intervalo de confianza; DG: diabetes gestacional; HIE: hipertensión inducida por el embarazo.

Tabla 2: Antecedentes familiares y obstétricos.

\section{DISCUSIÓNY CONCLUSIONES}

Es importante destacar el peso relativo del antecedente familiar de diabetes alcanzado en la presente casuística $(55,3 \%)$, marcadamente superior al identificado en estudios previos desarrollados en nuestro medio ${ }^{15}$. El fenómeno guarda relación con el crecimiento de la prevalencia de diabetes reportada en la población general ${ }^{16}$.

Los antecedentes obstétricos patólogicos registrados (Tabla 1) muestran que las pacientes con DG reclutadas presentaron distintos factores de riesgo para el desarrollo de DG. Lamentablemente no todos ellos son prevenibles; en efecto, el impacto negativo de la edad ${ }^{17}$, los antecedentes familiares de diabetes ${ }^{18}$ y el hecho de haber desarrollado DG en embarazos previos ${ }^{19}$ no son modi- ficables y escapan al posible efecto del programa educativo del EduGest. Consecuentemente se analizaron aquellos sobre los cuales es posible actuar y que serían tributarios de nuestro programa.

En las personas inicialmente reclutadas para el EduGest, la obesidad estuvo presente en $26,2 \%$ de los casos, cifra inferior a la descripta anteriormente $^{15}$ y de lo reportado a nivel poblacional ${ }^{16}$. También se registró su presencia en el último trimestre de embarazo, tramo en el cual se encontraba la mayoría de estas embarazadas. En ese período las pacientes manifestaron dicho estatus según el criterio de Rosso-Mardones ${ }^{20}$. Este no es un dato menor dada la relación significativa demostrada entre el IMC prenatal y la macrosomía fetal, independientemente de la presencia de DG 
dado que es también un predictor de esta última en ausencia de DG materna ${ }^{21-23}$.

En nuestra población muestral, los niveles de TG medidos en el último trimestre del embarazo se ubicaron por encima del valor de corte normal14. La evidencia disponible en la literatura muestra que en mujeres con DG y sobrepeso/obesidad, el aumento del nivel de TG es parcialmente responsable de la macrosomía, aún en presencia de buen control de la glucemia materna durante la gestación ${ }^{24}$. De hecho, la hipertrigliceridemia materna al final de la gestación es un predictor independiente de macrosomía en mujeres sin diabetes ${ }^{25}$.

La presencia combinada de obesidad e hipertrigliceridemia registrada en la población EduGest representa un aumento del riesgo de macrosomía, con repercusión negativa tanto sobre la madre (riesgo de trabajo de parto prolongado, cesárea, hemorragia postparto y laceraciones vaginales) ${ }^{26}$ como sobre el recién nacido (riesgo de fractura de clavícula, lesiones del plexo braquial, hipoglicemia, hiperbilirrubinemia, dificultad respiratoria y miocardiopatía hipertrófica) ${ }^{27,28}$.

Estos resultados dan sustento a la posibilidad de implementar el EduGest dado que el período gestacional es una oportunidad para promover conductas saludables que repercutirían favorablemente a corto y largo plazo. Esta aseveración se apoya en la baja prevalencia de actividad física en mujeres jóvenes en general y la alta prevalencia de obesidad y diabetes en nuestra comunidad ${ }^{16}$. Complementariamente, dichas conductas serían también importantes para promover el aumento de la actividad física en mujeres en edad fértil antes, durante y después del embarazo ${ }^{29}$.

Dado que la mayoría de nuestras participantes hizo su consulta en torno a la tercera semana ${ }^{28}$, sería necesario intensificar las estrategias de difusión del EduGest en la comunidad para lograr que dicha consulta ocurra al comienzo del embarazo, lo cual permitiría desarrollar el programa educativo en tiempo y forma.

En conclusión, la DG es un problema de salud pública en rápido crecimiento que se asocia con resultados adversos a corto y largo plazo tanto para las madres como para el producto de la gestación, hecho que resalta la importancia de su prevención. Cabe además recordar que la DG genera un círculo vicioso entre la futura DM2 en la madre, y la obesidad y DM2 en el niño. La ruptura de este círculo vicioso es parte de los objetivos del EduGest.
El cambio de hábitos no saludables siempre es un reto; sin embargo, habitualmente las mujeres que enfrentan un embarazo están fuertemente motivadas para aceptarlo en función de mejorar los resultados del embarazo y la salud del recién nacido ${ }^{30}$.

\section{Fuentes de financiamiento}

La implementación del EduGest fue parcialmente financiada por la Fundación Mundial de Diabetes (World Diabetes Foundation, WDF 15-1314).

\section{Agradecimientos}

Los autores agradecen la eficiente y generosa cooperación de todo el personal de la Facultad de Medicina de la UNNE y de la Municipalidad de la Ciudad de Corrientes. Igualmente agradecen la cooperación brindada por el Sr. Decano de la Facultad de Medicina de la UNNE, Profesor Mag. Omar Larroza, y las contadoras Dana Zimmerman y Analia Falcón de la misma Facultad. También agradecen la valiosa colaboración del Dr. Enzo Rucci por el desarrollo del sistema informático que administra la carga y el análisis de la base de datos. Jorge F. Elgart y Juan J. Gagliardino son miembros de la Carrera del Investigador del CONICET.

\section{BIBLIOGRAFÍA}

1. Jovanovic L, Pettitt DJ. Gestational diabetes mellitus. JAMA 2001; 286:2516-8.

2. Buchanan TA, Xiang AH. Gestational diabetes mellitus. J Clin Invest 2005; 115:485-91.

3. Reece EA, Leguizamon G, Wiznitzer A. Gestational diabetes: the need for a common ground. Lancet 2009; 373:1789-97.

4. Hiperglucemia y embarazo en las Américas: informe final de la Conferencia Panamericana sobre Diabetes y Embarazo (Lima, Perú. 8-10 de setiembre del 2015). Washington, DC:OPS; 2016. ISBN 978-92-75-31883-6.

5. CDC. National Diabetes Fact Sheet: National estimates and general information on diabetes and prediabetes in the United States. Atlanta, Ga, USA: Centers for Disease Control and Prevention; 2011. (Edited by C.f.D.C.a.P. Department of Health and Human Services).

6. Phillips PJ, Jeffries B. Gestational diabetes, worth finding and actively treating. Aust Fam Physician 2006; 35:701-3.

7. Lee AJ, Hiscock RJ, Wein P, Walker SP, Permezel M. Gestational diabetes mellitus: clinical predictors and long-term risk of developing type 2 diabetes: a retrospective cohort study using survival analysis. Diabetes Care 2007; 30:878-83.

8. Brown J, Alwan NA, West J, Brown S, McKinlay CJ, Farrar $D$, et al. Lifestyle interventions for the treatment of women with gestational diabetes. Cochrane Database Syst Rev 2017; 5:CD011970. doi: 10.1002/14651858.CD011970.pub2.

9. Brown J, Grzeskowiak L, Williamson K, Downie MR, Crowther CA. Insulin for the treatment of women with gestational diabetes. Cochrane Database Syst Rev 2017; 11. CD012037. doi: 10.1002/14651858.CD012037.pub2. 
10. WHO. Mastering depression in Primary Care. Info package. Frederisborg: World Health Organization, Regional Office for Europe, Psychiatric Research Unit, 1998.

11. Lowe B, Spitzer RL, Grafe K, Kroenke K, Quenter A, Zipfel S, et al. Comparative validity of three screening questionnaires for DSM-IV depressive disorders and physicians' diagnoses. J Affect Disord 2004; 78:131-40.

12. Guías de diagnóstico y tratamiento de la diabetes gestacional. ALAD 2016. Rev ALAD 2016; 6:155-69.

13. García SM, Lapertosa S, Rucci E, Arias V, Fasano MV, Kronsbein P. Nutriquid-Gest: cuestionario estructurado y autoadministrado para evaluar la ingesta alimentaria en mujeres embarazadas. Validación de una encuesta alimentaria. Rev ALAD 2019; 9:31-43.

14. Ywaskewycz LBG, Castillo MS, López D, Pedrozo W. Perfil lipídico por trimestre de gestación de una población de mujeres adultas. Rev Chil Obstet Ginecol 2010; 75:227-33.

15. Etchegoyen GS, de Martini ER, Parral Longobardi C, Cédola N, Alvariñas J, González C, et al. Diabetes gestacional: determinación del peso relativo de sus factores de riesgo. Medicina (Buenos Aires) 2011; 61:161-66.

16. Cuarta Encuesta Nacional de Factores de Riesgo. Resultados preliminares. $1^{\circ} \mathrm{Ed}$. Ciudad Autónoma de Buenos Aires: Instituto Nacional de Estadísticas y Censos (INDEC), Secretaría de Gobierno de Salud 2019. ISBN 978-950-896-542-4.

17. Pinheiro RL, Areia AL, Mota Pinto A, Donato H. Advanced maternal age: adverse outcomes of pregnancy, a meta-analysis. Acta Med Port 2019; 32:219-26.

18. Moosazadeh M, Asemi Z, Lankarani KB, Tabrizi R, Maharlouei N, Naghibzadeh-Tahami A, et al. Family history of diabetes and the risk of gestational diabetes mellitus in Iran: a systematic review and meta-analysis. Diabetes Metab Syndr 2017; 11(Suppl.1):S99-S104.

19. Schwartz N, Nachum Z, Green MS. The prevalence of gestational diabetes mellitus recurrence effect of ethnicity and parity: a metaanalysis. Am J Obstet Gynecol 2015; 213:310-17.
20. Mardones F, Rosso P. A weight gain chart for pregnant women designed in Chile. Matern Child Nutr 2005; 1(2):77-90.

21. Ehremberg HM, Mercer BM, Catalamo PM. The influence of obesity and diabetes on the prevalence of macrosomia. Am J Obstet Gynecol 2004; 191:964-8.

22. Ricart W, López J, Mozas J, Pericot A, Sancho MA, González N, et al. Body mass index has a greater impact on pregnancy outcomes than gestational hyperglycaemia. Diabetologia 2005; 48:1736-42.

23. Owens LA, O'Sullivan EP, Kirwan B, Ávalos G, Gaffney G, Dunne F; ATLANTIC DIP collaborators. ATLANTIC DIP: the impact of obesity on pregnancy outcome in glucose-tolerant women. Diabetes Care 2010; 33(3):577-9.

24. Olmos PR, Rigotti A, Busso D, Berkowitz L, Santos JL, Borzone $\mathrm{GR}$, et al. Maternal hypertriglyceridemia: a link between maternal overweight-obesity and macrosomia in gestational diabetes. Obesity (Silver Spring) 2014; 22:2156-63.

25. Wang X, Guan Q, Zhao J, Yang F, Yuan Z, Yin Y, et al. Association of maternal serum lipids at late gestation with the risk of neonatal macrosomia in women without diabetes mellitus. Lipids Health Dis $2018 ; 17: 78$.

26. Zhang X, Decker A, Platt RW, Kramer MS. How big is too big? The perinatal consequences of macrosomia. Am J Obstet Gynecol 2008; 198:517.

27. Merzouk $\mathrm{H}$, Khan NA. Implication of lipids in macrosomia of diabetic pregnancy: can $n-3$ polynsaturated fatty acids exert beneficial effects? Clin Sci (Lond) 2003; 105:519-29.

28. Chahuan SP, Grobman WA, Gherman RA, Chahuan VB, Chang $\mathrm{G}$, Magann EF, et al. Suspicion and treatment of the macrosomic fetus: a review. Am J Obstet Gynecol 2005; 193:332-4.

29. Dipietro L, Evenson KR, Bloodgood B, Sprow K, Troiano RP, Piercy KL, et al. Physical Activity Guidelines Advisory Committee. Benefits of physical activity during pregnancy and postpartum: an umbrella review. Med Sci Sports Exerc 2019; 51:1292-1302.

30. Phelan S. Pregnancy: a "teachable moment" for weight control and obesity prevention. Am J Obstet Gynecol 2010; 202:e131-e138.

\section{APÉNDICE}

Las siguientes instituciones y personas son miembros del EduGest en las diferentes CAPs, maternidades y hospitales, responsables de la atención médica de la población del programa. - Hospital Ángela Iglesia de Llano, Corrientes: Rivero Mabel Itatí; Álvarez Spence Stella María; Fernández, Claudia; González Rosa; López Claudio; Rebollo Cecilia Yanina; Vallejos Basterra María Clara. - Hospital José Ramón Vidal, Corrientes: Ludman Verónica; Bertoli Sandra; Bordón Gabriel; Gutiérrez Juan Pablo; Montenegro Alejandra; Veglia Valentina.

- Hospital General de Agudos Dr. Cosme Argerich, CABA: Mezzabotta Leonardo; Barrios Etelvina; Burgos Guillermina; Bustos María Virginia; Camín Paula; Del Campo María José; Dominik Elida; Hidalgo Gisela Elizabeth; Maldonado Griselda; Nasello Macarena; Pla María Florencia; Zafrán Eliana.

- Maternidad Eloísa Torrent de Vidal, Corrientes: de Sagastizabal Teresa; Aguirre Daniel; Cardozo Alberto; Gómez Norma Beatriz; Morales Elba; Pérez Marcela Alejandrina.

- Hospital Municipal Materno Infantil, San Isidro, provincia de Buenos Aires: Rodríguez María Elena; Covelli Daniela; Miraglia Sofía.

- Hospital General de Agudos Dr. José Ramos Mejía, CABA: Bragagnolo Julio; Castelli Fernando; Galarza Natalia; Houssay Solange; López Myriam; Ortensi Graciela; Redolfi Belén; Vanoli Guadalupe.
- Hospital Zonal Dr. Camilo Muniagurria, Goya, Corrientes: Orrego Miriam Teresita; Bernasconi Marta; De La Vega Ana; Ebel Silvana; Fiorito Laura; Ganancia Pilar; Martínez David; Suñé Lourdes.

- Hospital Provincial Dr. Fernando Irastorza, Curuzú Cuatiá, Corrientes: González Mónica; Alaris Rosa delValle; Estigarribia Silvina; Gómez María Rosa; Martínez Rocío; Silva Diego; Zucco Anabella. - Hospital Perrando, Resistencia, Chaco: Candia Patricia; Achitte Eduardo; Brollo Camila; Chandia Cecilia Inés; Chaves Caballero María Elena; Gilberto Olga Liliana; González Alicia del Carmen; Maldonado José; Morzán Ana Carolina; Roldán Itatí.

- Hospital Donación Francisco Santojanni CABA: Oviedo Alejandra; Arakaki Haydee; Fiore Eduardo; Gandolfi Lucas; Geronazzo Verónica; Kusevitzky Raquel; Orrigo Carolina.

- Hospital Municipal Dr. Diego Thompson, San Martín, provincia de Buenos Aires: Suárez Cordo Cristian; Cruz María José; Kos Mirian Verónica; Sznaider Daniel Alberto.

- Facultad de Medicina, Universidad Nacional del Nordeste, Corrientes: Arias Tichij Valeria; Costa Dana Jazmín; Demuth Mercado Patricia Belén; Fernández Graciela; Romero Vidomlansky Patricia Ruth; Rovira Gabriela; Sotelo Nancy Edda.

- Municipalidad de la Ciudad de Corrientes: Alegre Hilda; Aquino Clara; Burgos Beatriz; Lombardi María Silvana; Simón Victoria; Tabozzi Silvia. 